\title{
Permissible Observability Relations in FSM Networks *
}

\author{
Huey-Yih Wang Robert K. Brayton \\ Department of EECS, University of California, Berkeley, CA 94720
}

\begin{abstract}
Previous attempts to capture the phenomenon of output don't care sequences for a component in an FSM network have been incomplete. We demonstrate that output don't care sequences for a component can be expressed using a set of observability relations given that its state transition function is kept unchanged. Each observability relation is permissible in the sense that any implementation compatible with one of them is feasible. The representation for a set of permissible observability relations is not unique. We provide a method to find a set with the minimum number of permissible relations. We briefly discuss the exploitation of permissible observability relations in state minimization, circuit implementation and signal encoding. We have implemented these methods and present some preliminary results on a few small artificially constructed examples.
\end{abstract}

\section{Introduction}

The flexibility in implementing an isolated combinational logic circuit can be expressed by don't cares. For an individual component in a hierarchically designed combinational logic circuit, a Boolean relation, an observability relation or a symbolic relation is required to express all possible implementations $[3,1,10,17]$. This freedom in implementation is due to reduced controllability and observability from the environment. By exploiting this information, we often can achieve a better implementation for that component.

Similarly, sequential don't cares are important in the optimization of sequential circuits. Several approaches have been proposed. For example, in [11], unreachable states and equivalent states are exploited in the logic optimization of an isolated sequential circuit. Damiani et al. [5] introduced the notion of synchronous relations to deal with the logic optimization of pipelined sequential circuits. This approach is motivated by a circuit implementation point of view. On the other hand, a transition relation can be used to represent an isolated finite state machine (FSM). This allows us to deal with symbolic information, i.e. with unencoded machines. Incompletely specified information often refers to possible implementations. Exploiting this information may also change the state minimality of a machine. In general, a transition relation can be regarded as an observability relation or a symbolic relation. State minimization for an isolated machine has been well studied $[13,8]$. Although state minimality does not imply that the resultant logic circuit after state encoding is minimized, it is generally regarded as a good starting point for state encoding to get smaller logic implementations.

In the case of sequential don't cares for an individual component in a network of FSM's, ${ }^{1}$ we may need to consider sequences of don't cares. There are a few studies related to this problem $[9,6,14,19]$. Although by flattening a network of FSM's into a composite machine we may perform global optimization, the composite machine is often too big to be handled by synthesis tools. To perform hierarchical synthesis, we must consider the interaction between components. The computation and exploitation of don't care information is crucial for the quality of the resultant circuit implementation.

The computation of don't care information for a component in an FSM network is much harder than its counterpart in combinational logic. We divide this problem into two parts: sequential input and

\footnotetext{
* This project was supported by DARPA under contract number JFBI90-073 and NSF under contract number EMC-84-19744 and MIP-87-19546.

${ }^{1}$ In this paper, only synchronous FSM networks with known initial states are considered.
}

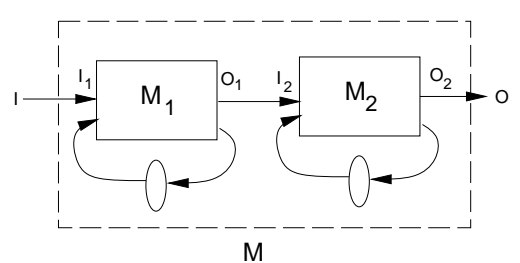

Figure 1: A cascade circuit of two FSM's.

output don't cares. In this paper, we deal with the latter. Consider the cascade machine in Figure 1. The flexibility in implementing $M_{1}$ when cascaded with $M_{2}$ is called sequential output don't cares. This was studied by Devadas [6], and later by Rho et al. [14] who generalized Devadas' procedure to compute fixed-length output don't care sequences.

In this paper, after reviewing previous work in section 3 , we explain why the notion of information lossyness introduced in [14] can not completely characterize the phenomenon of output don't care sequences. Then, we discuss the difficulties in computing and expressing these. We demonstrate that output don't care sequences for a component can be expressed using a set of observability relations given that its state transition function is kept unchanged. In section 5, we propose an implicit enumeration algorithm which exactly computes them. Each observability relation is permissible in the sense that the behavior of the network is preserved. We describe how to exploit them in state minimization, circuit implementation and signal encoding. Finally, we give some preliminary results on some artificially constructed circuits.

\section{Preliminaries}

\subsection{Finite Automata and Finite State Machines}

A deterministic finite automaton (DFA), $\mathcal{A}$, is a quintuple $(K, \Sigma, \delta$, $\left.q_{0}, F\right)$ where $K$ is a finite set of states, $\Sigma$ an alphabet, $q_{0} \in K$ the initial state, $F \subseteq K$ the set of final states, and $\delta$ the transition function, $\delta: K \times \Sigma \rightarrow K$. A nondeterministic finite automaton (NFA), $\mathcal{A}$, is a quintuple $\left(K, \Sigma, \delta, q_{0}, F\right)$ where $\delta$, the transition relation, is a finite subset of $K \times \Sigma^{*} \times K$, and $\Sigma^{*}$ the set of all strings obtained by concatenating zero or more symbols from $\Sigma$. An input string is accepted by $\mathcal{A}$ if it ends up in one of final states of $\mathcal{A}$. The language accepted by $\mathcal{A}, \mathcal{L}(\mathcal{A})$, is the set of strings it accepts.

A finite state machine (FSM), $M$, is a six-tuple $\left(I, O, Q, \delta, \lambda, q_{0}\right)$, where $I$ is a finite input alphabet, $O$ a finite output alphabet, $Q$ a finite set of states, $\delta$ the transition function, $\lambda$ the output function, and $q_{0}$ the initial state. A machine is of Moore type if $\lambda$ does not depend on the inputs, and Mealy otherwise. An FSM can be represented by a state transition graph (STG). A machine in which transitions under all input symbols from every state are defined is a completely specified machine; in other words, both $\delta$ and $\lambda$ are complete functions. Otherwise, a machine is incompletely specified.

A distinguishing sequence for two states $q_{1}, q_{2} \in Q$ is a sequence of inputs such that when applied to $M$, the last input produces different outputs depending whether $M$ started at $q_{1}$ or $q_{2}$. In a completely specified machine $M$, two states $q_{1}$ and $q_{2}$ are equivalent if there is no distinguishing sequence. In an incompletely specified machine $M$, two such states $q_{1}$ and $q_{2}$ are compatible.

A cascade of FSM's $M_{1}$ and $M_{2}$, denoted $M_{1} \rightarrow M_{2}$, is shown in 


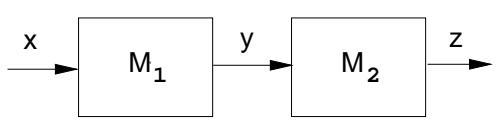

Figure 2: A cascade of two combinational circuits.

Figure 1. $M_{1}$ is called the driving machine, $M_{2}$ the driven machine. For $x \in Q_{M_{1}}$, its co-reachable states in $M_{2}$ are $\left\{y \mid y \in Q_{M_{2}}\right.$ such that $(x, y)$ is a reachable state in the cascade machine $\left.M_{1} \rightarrow M_{2}\right\}$. Similarly, a state in $M_{2}$ has co-reachable states in $M_{1}$.

\subsection{Set Computation and Operators}

Let $B$ designate the set $\{0,1\}$.

Definition 1 Let $E$ be a set and $S \subseteq E$. The characteristic function of $S$ is the function $\chi_{S}: E \rightarrow B$ defined by $\chi_{S}(x)=1$ if $x \in S$, and $\chi_{S}(x)=0$, otherwise.

Definition 2 Let $f: B^{n} \rightarrow B$ be a Boolean function, and $x=$ $\left\{x_{1}, \ldots, x_{k}\right\}$ a subset of the input variables. The existential quantification (smoothing) of $f$ by $x$, with $f_{a}$ denoting the cofactor of $f$ by literal a is defined as :

$$
\begin{aligned}
\exists_{x_{i}} f & =f_{x_{i}}+f_{\overline{x_{i}}} \\
\exists_{x} f & =\exists_{x_{1}} \ldots \exists_{x_{k}} f .
\end{aligned}
$$

Definition 3 Let $f: B^{n} \rightarrow B$ be a Booleanfunction, only depending on a subset of variables $y=\left\{y_{1}, \ldots, y_{k}\right\}$. Let $x=\left\{x_{1}, \ldots, x_{k}\right\}$ be another subset of variables, describing another subspace of $B^{n}$ of the same dimension. The substitution of variables $y$ by variables $x$ in $f$ is the function of $x$ obtained by substituting $x_{i}$ for $y_{i}$ in $f$

$$
\left(\theta_{y, x} f\right)(y)=f(x) \text { if } x_{i}=y_{i} \text { for all } 1 \leq i \leq k .
$$

Reduced ordered binary decision diagrams (BDD's) [2] are well suited to represent the characteristic functions of subsets of a set, and efficient algorithms exist to manipulate them to perform all standard Boolean operations. As a result, the above set operations can be done efficiently.

The reachable states can be computed efficiently using implicit state enumeration techniques introduced by Coudert et al. [4]. This approach is based on representing a set of states by a characteristic function which can be manipulated effectively using BDD's. In the following, we represent an FSM implicitly by a characteristic function using BDD's.

Definition 4 The transition relation of an FSM $M=(I, O, Q, \delta, \lambda$, $\left.q_{0}\right)$ is a function $T: I \times Q \times Q \times O \rightarrow B$ such that $T(i, p, n, o)=1$ if and only if state $n$ can be reached in one state transition from state $p$ and produce output o when input $i$ is applied.

\section{Previous Work}

\subsection{Observability Relation for Combinational Logic}

In a hierarchically designed combinational logic circuit, all possible implementations can be represented by a single Boolean relation, an observability relation or a symbolic relation ${ }^{2}[3,1,10,17]$. For example, as shown in Figure $2, M$ is a cascade machine $M_{1} \rightarrow$ $M_{2}$, where $M_{1}$ and $M_{2}$ are combinational circuits. Let $\mathcal{O}(x, z)$, $\mathcal{O}_{1}(x, y)$, and $\mathcal{O}_{2}(y, z)$ be the observability relations of $M, M_{1}$, and $M_{2}$, respectively. If $\mathcal{O}(x, z)$ and $\mathcal{O}_{2}(y, z)$ are given, $\mathcal{O}_{1}(x, y)$ can be computed as follows :

$$
\mathcal{O}_{1}(x, y)=\exists_{z}\left(\mathcal{O}(x, z) \cdot \mathcal{O}_{2}(y, z)\right) .
$$

$\mathcal{O}_{1}(x, y)$ captures all possible implementations of $M_{1}$ without violating the desired behavior of the cascade machine $M$. Exploiting this freedom in implementation often leads to better logic implementations.

${ }^{2}$ In this paper, we will not make a distinction among Boolean relations, observability relations and symbolic relations unless necessary.

\subsection{Sequential Output Don't Cares}

If $M_{1}$ and $M_{2}$ in Figure 2 are FSM's, computing the flexibility in implementing $M_{1}$ is much harder. Devadas [6] addressed this problem as computing sequential output don't cares for $M_{1}$ and proposed a simple heuristic to compute partial don't care information for $M_{1}$. Consider a transition edge $e$ in the STG of $M_{1}$. Let the output symbol of $e$ be $v_{1}$. Devadas' procedure first computes the co-reachable states in $M_{2}$ corresponding to the present state of transition $\epsilon$. If for every corresponding co-reachable state in $M_{2}$, an output symbol $v_{2}$ from $M_{1}$ drives machine $M_{2}$ to produce the same output and next state as the original output symbol $v_{1}$ from $M_{1}$ does, then the output part $\left\{v_{1}\right\}$ of $\epsilon$ is expanded to $\left\{v_{1}, v_{2}\right\}$. This is repeated on $e$ for each output symbol $v_{2}$ of $M_{1}$. Then the above process is repeated for each transition edge $e$ in the STG of $M_{1}$. This output expansion procedure does not change state reachability of the composite machine $M_{1} \rightarrow M_{2}$.

This procedure, in fact, is restrictive ${ }^{3}$. First, it considers only one transition edge of $M_{1}$ at a time, and excludes the possibility of simultaneous output expansions among all transition edges. That is, an expanded output symbol in a transition edge may depend on the expanded output symbols of the other transition edges. Furthermore, next states need not be the same when an output expansion takes place. To be more specific, the set of co-reachable states for each state in $M_{1}$ may change due to simultaneous output expansions. In the next subsection, we show this by example.

\subsection{Output Don't Care Sequences}

Later, Rho et al. [14] considered sequences of output don't cares and used the notion of information lossyness to explain the phenomenon of these sequences in a cascade machine. A machine is said to be information lossless if given the initial state, the final state and the output sequence, the corresponding input sequence can be uniquely determined. A machine that is not lossless is said to be lossy. A state $s$ of a machine is said to be a lossy state if starting from $s$ there exist two distinct finite-length input sequences such that their output sequences and final states are the same. An information lossless machine cannot contain any lossy states.

Consider the cascade machine $M_{1} \rightarrow M_{2}$ in Figure 1. Rho et al. [14] interpreted that output don't care sequences for $M_{1}$ are due to the lossyness of $M_{2}$. Based on this explanation, if $M_{2}$ has no lossy states, there are no output don't care sequences for $M_{1}$. In this sense, only lossy states in $M_{2}$ need to be considered for computing output don't care sequences. Accordingly, they gave the following definitions for equivalent sequences and equivalent machines. Input sequences that lead a driven machine $M_{2}$ from the same initial state $s$ to the same final state $t$ and produce the same output sequences, are said to be equivalent with respect to state $s$. Thus, state $s$ is a lossy state. Two machines $M_{1}^{\prime}$ and $M_{1}^{\prime \prime}$ are said to be equivalent with respect to $M_{2}$ if and only if for each input sequence they produce output sequences that are in the same equivalence class of input sequences of $M_{2}$, i.e. ending in the same final state if $M_{2}$ is state-minimal.

According to the above definitions, a heuristic was proposed in [14] to compute a subset of fixed-length output don't care sequences for $M_{1}$. This is an extension of Devadas' procedure [6]. To compute fixed-length, say $k$-length, equivalent sequences in $M_{2}$, this procedure first unrolls $M_{1}$ and $M_{2}$ for $k$-length time frames. Let the unrolled machines of $M_{1}$ and $M_{2}$ be $M_{1}^{k}$ and $M_{2}^{k}$, respectively. An unrolled machine has the same state space as that of the original machine except that for each transition edge the input part is a $k$-length input sequence, and the output a $k$-length output sequence. By such a construction, $k$-length equivalent sequences starting at a state $s$ of $M_{2}^{k}$ can be computed. In contrast, Devadas' procedure computes equivalent value from a state $s$ in $M_{2}$. Then the output part of each transition edge in $M_{1}^{k}$ can be expanded using the same rationale in Devadas' procedure except that a consistency check needs to be performed between the input and output part of all transition edges in $M_{1}^{k}$. Finally, to construct a non-unrolled machine of $M_{1}^{k}, M_{1}{ }^{\prime}$, a heuristic based on state splitting may be employed to accommodate this don't care information as

${ }^{3}$ This is in contrast to the comment, "This is not restrictive, as long as we can assume that $M_{2}$ is state-minimal.", made in [15]. 

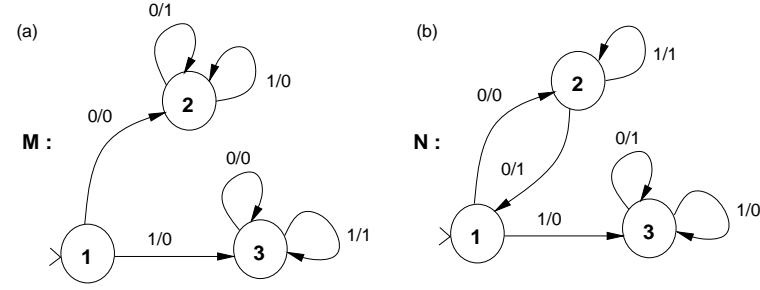

Figure 3: (a) An information lossless and state-minimal machine. A lossy and state-minimal machine.

much as possible. This procedure explicitly enumerates fixed-length output don't care sequences. In general, output don't care sequences are of infinite length. The complexity of this procedure may grow exponentially with the length of don't care sequences.

This procedure is an elegant extension of [6]. However, their interpretation of output don't care sequences is not general. By their definition, output don't care sequences for $M_{1}$ can be interpreted as those input sequences starting from the initial state of $M_{2}$ (assumed to be lossy and state-minimal), producing the same output sequence, and ending in the same final state. Their reasoning is as follows. If the output sequence of $M_{2}$ is altered, so is the overall behavior of the cascade. Also, if the final state is different, the behavior of the cascade changes, unless the new final state is equivalent to the original one.

There are several factors not considered in this reasoning.

First, output sequences from $M_{1}$ may change the equivalence of states in $M_{2}$ simultaneously, since the complement of output sequences from $M_{1}$ are input don't care sequences for $M_{2}$, which may change the state minimality of $M_{2}$ [9]. During the process of exploiting output don't care sequences for $M_{1}$, the output function of $M_{1}$ is changed at the same time. Consequently, we are unable to assume that state minimality of $M_{2}$ is invariant.

Secondly, by definition, different input sequences applied to an information lossless machine may produce the same output sequences, but end in different final states. Consequently, if $M_{2}$ produces the same output sequences for different input sequences, it is not necessarily lossy. In other words, there might exist output don't care sequences even if $M_{2}$ is information lossless. For example, machine $M$ in Figure $3(a)$ is information lossless and state-minimal. Input sequences $\left(11^{*}\right)$ and $\left(00^{*}\right)$ produce the same output sequences $\left(01^{*}\right)$, but do not end in the same final state. As a consequence, it is not necessary that input sequences which produce the same output sequences end in the same final state even when the driven machine is state-minimal. Moreover, this argument is not valid even for a lossy and state-minimal machine. An example is machine $N$ shown in Figure 3(b). $N$ is lossy since state 1 is a lossy state ${ }^{4}$. Input sequences $(00)^{*}$ and $(10)^{*}$ produce the same output sequences $(01)^{*}$, but do not end in the same final state.

Consider the cascade machine $M \equiv M_{1} \rightarrow M_{2}$ as shown in Figure 4. This is an example where there are output don't care sequences for $M_{1}$ even when $M_{2}$ is state-minimal and information lossless. Let $v_{i}$ denote the output value of a transition edge $\epsilon_{i}$ in the STG of $M_{1}$. The value of $\left(v_{1}, v_{2}, v_{3}, v_{4}, v_{5}, v_{6}\right)$ in Figure 4 is $(0,1,0,1,0,1)$. Since the output sequences of $M_{1}$ are $\{0,1\}^{*}$, there are no input don't care sequences for $M_{2}$. Thus, we cannot use any input don't care information to simplify $M_{2}$ first. If we apply Devadas' [6] or Rho's [14] procedures, we cannot find any sequential output don't cares for $M_{1}$. However, any one of the following values of $\left(v_{1}, v_{2}, v_{3}, v_{4}, v_{5}, v_{6}\right)$ preserves the same behavior as $M_{1} \rightarrow M_{2}$.

$$
\left(v_{1}, v_{2}, v_{3}, v_{4}, v_{5}, v_{6}\right)=\left\{\begin{array}{l}
(0,1,0,1,0,1) \\
(0,1,0,0,1,0) \\
(1,0,1,0,1,0) \\
(1,0,1,1,0,1)
\end{array}\right.
$$

${ }^{4}$ Input sequences 0010,1010 from initial state 1 produce the same output sequence 0101 , and end in the same state 3 .
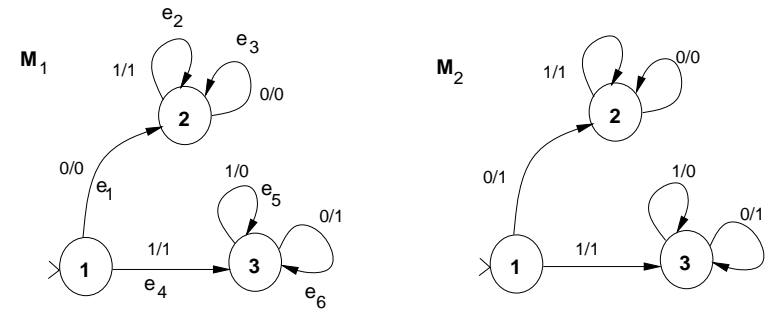

Figure 4: An example: $M \equiv M_{1} \rightarrow M_{2}$, where both $M_{1}$ and $M_{2}$ are state-minimal and information lossless.

The reachable states of $M_{2}$ are $\{1,2,3\}$ when $\left(v_{1}, v_{2}, v_{3}, v_{4}, v_{5}, v_{6}\right)=$ $(0,1,0,1,0,1)$. But when $\left(v_{1}, v_{2}, v_{3}, v_{4}, v_{5}, v_{6}\right)=(0,1,0,0,1,0)$, the reachable states of $M_{2}$ are $\{1,2\}$. So, the state reachability may change when we have different output sequences from $M_{1}$. This is why Devadas' procedure is restrictive.

Consequently, the previous definitions of machine equivalence for the driving machine do not include all possible machines which when cascaded by $M_{2}$ produce the same behavior as the original cascade machine. As a matter of fact, the general definition of machine equivalence should be the following : two machines $M_{1}{ }^{\prime}$ and $M_{1}{ }^{\prime \prime}$ are equivalent with respect to $M_{2}$ if and only if $M_{1}{ }^{\prime} \rightarrow M_{2}$ and $M_{1}{ }^{\prime \prime} \rightarrow M_{2}$ have the same input/output behavior. This specifies the full flexibility for implementing $M_{1}$. Therefore, this should be regarded as the general definition of sequential output don't cares.

\section{Permissible Observability Relations}

As mentioned earlier, an incompletely specified FSM can be expressed by a symbolic relation. Using this representation for an isolated FSM, two kinds of don't care information can be conveyed. The first is known as input-incompletely-specified don't cares or unspecified transitions. They characterize the situation that a given input symbol never occurs when a machine is in a particular state, since there are limited kinds of sequences that can be applied to the machine. The other kind is called output-incompletely-specified don't cares. They occur when we are not interested in an output symbol associated with a given state or state transition. In the following, we investigate whether this representation is powerful enough to convey sequential don't cares for a component in an interacting FSM network.

In a cascade circuit $M_{1} \rightarrow M_{2}$ as shown in Figure 1, we can compute input don't cares sequences for $M_{2}$ by keeping $M_{1}$ unchanged. The general procedure known to solve this problem is due to Kim and Newborn [9]. This procedure summarizes output sequences from $M_{1}$ by an NFA $\mathcal{A}^{\prime}$, and then transforms $\mathcal{A}^{\prime}$ into a minimal DFA $\mathcal{A}$. The equivalent machine to $M_{2}$ with input don't care sequences is the product machine $\mathcal{A} \times M_{2}$. The input don't cares sequences of $M_{2}$ are unspecified transitions in the resultant product machine. This product machine captures all input don't cares sequences, and we can represent it as an incompletely specified FSM. As a consequence, a single observability relation is sufficient to implicitly express input don't care sequences.

On the other hand, a single observability relation may not be sufficient to express output don't care sequences. We can compute output don't care sequences for $M_{1}$ by keeping $M_{2}$ unchanged. An FSM can be regarded as a language transducer, i.e. transforming a regular language to another regular language. Therefore, output sequences from $M_{1}$ can be expressed by a regular language, say $\mathcal{L}\left(M_{1}^{\circ}\right)$. We can define an equivalence class of languages with respect to $M_{2}$, $\left[\mathcal{L}\left(M_{1}^{\circ}\right)\right]_{M_{2}}$, such that any language in this equivalence class can be generated by a certain machine $M_{1}^{\prime}$ which preserves the same behavior as $M_{1} \rightarrow M_{2}$ when cascaded with $M_{2}$. Next, we explain the intrinsic difficulties in computing sequential output don't cares even when we adopt the definition of machine equivalence from [14], i.e. equivalent input sequences end up in the same final state if $M_{2}$ is state-minimal in isolation. Rho's procedure [14] computes fixed-length equivalent sequences, and then expands these to be output don't care sequences 


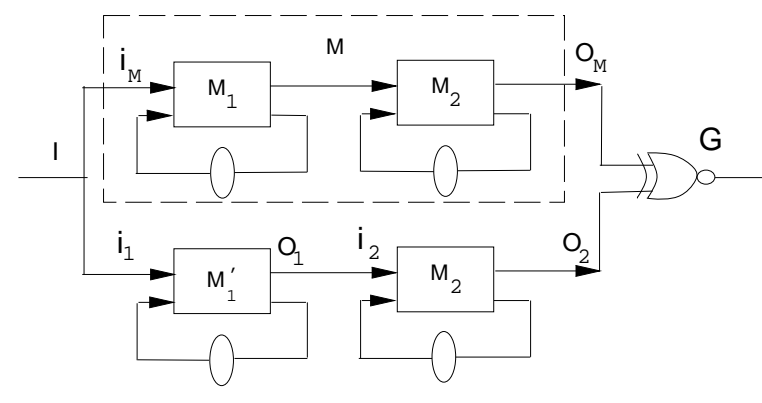

Figure 5: Feasibility testing using FSM equivalence checking.

for $M_{1}$. Exploiting this information, some equivalent machines may be derived. Let $M_{1}^{\prime}$ be an equivalent machine. Then $\mathcal{L}\left(M_{1}^{\prime o}\right)$, output sequences from $M_{1}{ }^{\prime}$, is in $\left[\mathcal{L}\left(M_{1}^{\circ}\right)\right]_{M_{2}}$. However, the length of equivalent sequences may be arbitrarily large. Furthermore, the complexity of this computation may grow exponentially with the length of output don't care sequences. Therefore, it is hard to enumerate all languages in $\left[\mathcal{L}\left(M_{1}^{\circ}\right)\right]_{M_{2}}$.

From another point of view, we can enumerate all possible languages produced by $M_{1}$ with its state transition function unchanged. Let the cardinality of the transition edges of $M_{1}$ be $k$, and that of the output alphabet of $M_{1}$ be $m$. If we keep the state transition function of $M_{1}$ unchanged, there are $m^{k}$ possible output functions, i.e. $m^{k}$ possible regular languages may be produced by $M_{1}$ (some of them may be the same). For each output function $\lambda_{1}{ }^{\prime}$, there is a corresponding machine $M_{1}^{\prime}$. The feasibility of $\lambda_{1}{ }^{\prime}$ can be checked by testing if $M_{1}{ }^{\prime} \rightarrow M_{2}$ preserves the same behavior as $M_{1} \rightarrow M_{2}$. This is shown in Figure 5. Using this naive approach, we can check all possible output functions one by one to find all feasible solutions. We may need $m^{k}$ invocations of FSM equivalence checking.

We can interpret the above approach as simultaneous output expansions among all transition edges. An expanded output symbol in a transition edge may be dependent on the expanded output symbols of other transition edges. Consequently, the flexibility of implementations captured by the above approach is more than for outputincompletely-specified don't cares in an isolated FSM. All feasible output functions possibly may not be expressed by a single observability relation. In fact, a set of observability relations is needed. We show this in the next section. Each observability relation is permissible in the sense that any output function compatible with one of the observability relations corresponds to a possible implementation.

In the next section, we present an implicit algorithm which finds all such feasible output functions by executing FSM equivalence checking once.

\section{Computation of Permissible Observability Rela- tions}

Let $M_{1}=\left(I_{1}, O_{1}, Q_{1}, \delta_{1}, \lambda_{1}, q_{1_{0}}\right)$ and $M_{2}=\left(I_{2}, O_{2}, Q_{2}, \delta_{2}, \lambda_{2}, q_{2_{0}}\right)$ be two FSM's. $M=\left(I, O, Q, \delta, \lambda, q_{M_{0}}\right)$ is $M_{1} \rightarrow M_{2}$, the cascade machine. Let $\Lambda_{1}$ denote the set of all possible output functions of $M_{1}$ while keeping its state transition function unchanged. Let $\left.M_{1}\right|_{\lambda_{1}{ }^{\prime}}$ denote a machine which is the same as $M_{1}$ except with an output function $\lambda_{1}{ }^{\prime} \in \Lambda_{1}$. Suppose that the cardinality of transition edges in $M_{1}$ is $k$. For each transition edge $e_{j}$, we associate it with a symbolic variable $v_{j}$ which takes values from $O_{1}$. Let $V$ denote the space spanned by $v_{0}, v_{1}, \ldots, v_{k-1}$, i.e. $O_{1}^{k}$. Any minterm in $V$ is called an output assignment. An output assignment corresponds to an output function in $\Lambda_{1}$, and vice versa. An assignment mapping is a bijective mapping $\mathcal{M}: V \rightarrow \Lambda_{1}$ which maps a minterm $v \in V$ to an output function $\lambda_{1}{ }^{\prime}$ in $\Lambda_{1}$. An output function $\lambda_{1}{ }^{\prime} \in \Lambda_{1}$ is feasible if and only if $\left.M_{1}\right|_{\lambda_{1}{ }^{\prime}} \rightarrow M_{2}$ preserves the same behavior as $M_{1} \rightarrow M_{2}$. An output assignment $v$ is feasible if and only if $\mathcal{M}(v)$ is a feasible output function. The set of feasible output assignments is denoted as

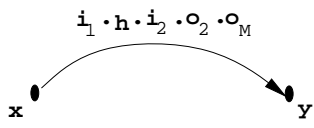

Figure 6: A transition defined in $T$.

$f(v)$, the feasible output assignment function.

Our goal is to compute all $\lambda_{1}{ }^{\prime} \in \Lambda_{1}$ such that $\left.M_{1}\right|_{\lambda_{1}{ }^{\prime}} \rightarrow M_{2}$ preserves the same behavior as $M_{1} \rightarrow M_{2}$. This is pictorially explained in Figure 5.

\subsection{Reachability Relation}

Here, we present an implicit enumeration method based on a generalization of implicit FSM equivalence checking. The most important step in the FSM equivalence checking is the computation of reachable states. The state space for our equivalence checking is $Q_{1} \times Q_{2} \times Q$, denoted as $S$. For an output assignment $v$, there is a corresponding output function $\mathcal{M}(v)$ and a set of reachable states which is a subset of $S$. Different output functions may result in different sets of reachable states. With this observation, we introduce the concept of reachability relation.

Definition $5 A$ reachability relation is a function $\mathcal{F}: S \times V \rightarrow B$ such that $\mathcal{F}(s, v)=1$ if and only if $s$ is reachable from the initial state when the output function is $\mathcal{M}(v)$.

For an output assignment $v$, the transition relation of $\left.M_{1}\right|_{\mathcal{M}(v)}$ is $\left.T_{1}\right|_{\mathcal{M}(v)}$. We can compose $\left.T_{1}\right|_{\mathcal{M}(v)}, T_{2}$, and $T_{M}$, and then use implicit reachability computation to check whether $G$ in Figure 5 is a tautology. However, this is an explicit enumeration method since we need to enumerate explicitly for all $v \in V$. To perform implicit enumeration, we construct an abstract transition relation for $M_{1}, T_{1}^{\prime}$, as follows. First, give a labeling for each transition edge in $M_{1}$. Let $h$ be a $k$-valued variable. We substitute $j$, i.e. the literal $h^{\{j\}}$, for the output part of $\epsilon_{j}$. The abstract transition relation is $T_{1}^{\prime}\left(i_{1}, p_{1}, n_{1}, h\right)$. Let the transition relation of $M_{2}$ be $T_{2}\left(i_{2}, p_{2}, n_{2}, o_{2}\right)$ and that of $M, T_{M}\left(i_{M}, p_{M}, n_{M}, o_{M}\right)$. Define $T\left(i, h, i_{2}, p, n, o_{2}, o_{M}\right)=T_{1}^{\prime} \cdot T_{2} \cdot T_{M}$, where $i=i_{1}=i_{M}$, $p=\left(p_{1}, p_{2}, p_{M}\right)$ and $n=\left(n_{1}, n_{2}, n_{M}\right)$. The initial state is $q_{0}=$ $\left(q_{10}, q_{2_{0}}, q_{M_{0}}\right)$. The motivation for constructing the abstract transition relation, $T_{1}^{\prime}$, is explained below. $T$ contains all possible transitions for any composite machine of $\left.M_{1}\right|_{\mathcal{M}(v)}, M_{2}$, and $M$. Consider a transition defined in $T\left(i, h, i_{2}, p, n, o_{2}, o_{M}\right)$ as shown in Figure 6. The expression associated with this transition is $\left(i \cdot h \cdot i_{2} \cdot o_{2} \cdot o_{M}\right)$. Let the value of $h$ be $j$. It means that when the output value of $e_{j}$ in $M_{1}$ is equal to that of $i_{2}$, this transition is made, and vice versa. Different output values of transition edge $e_{j}$ result in different output assignments. Therefore, this provides a way to relate output assignments to transitions in $T$.

Since $\mathcal{F} \subseteq S \times V, \mathcal{F}$ is a finite set. In the following theorem, we demonstrate that the reachability relation can be computed by a least fixed point computation.

Theorem 5.1 ${ }^{5}$ Let $\mathcal{P}(p, n, v)$ be defined as follows :

$$
\mathcal{P}(p, n, v)=\sum_{j=0}^{k-1} \theta_{i_{2}, v_{j}}\left\{\left[\left(\exists_{i, o_{2}, o_{M}} T\right)\right]_{(h=j)}\right\} .
$$

$\mathcal{F}(p, v)$ is the limit of the following sequence:

$$
\begin{aligned}
\mathcal{F}_{0} & =\left(p=q_{0}\right) \cdot 1 \\
\mathcal{F}_{m} & =\theta_{n, p} \exists_{p}\left\{\mathcal{F}_{m-1} \cdot \mathcal{P}(p, n, v)\right\}+\mathcal{F}_{m-1} \\
\mathcal{F}_{\infty} & =\mathcal{F}_{m} \text { if } \mathcal{F}_{m}=\mathcal{F}_{m+1} .
\end{aligned}
$$

${ }^{5}$ Detailed proofs of the theorems presented in this paper are given in [20]. 
Corollary 5.2 For an output assignment $\alpha$, its corresponding set of reachable states up to the $m$-th iteration $\left(R_{m}(p)\right)_{\alpha}$ is $\left(\mathcal{F}_{m}(p, v)\right)_{v=\alpha}$. In particular, the set of reachable states, $\left(R_{\infty}(p)\right)_{\alpha}$, is $\left(\mathcal{F}_{\infty}(p, v)\right)_{v=\alpha}$.

\subsection{Feasible Output Assignment Function}

An FSM can be regarded as a language transducer, i.e. transforming a regular language to another regular language. If $M_{2}$ is completely specified, $M_{2}$ maps $I_{2}{ }^{*}$ to another regular language. That is, for all input sequences, the behavior of $M_{2}$ is defined. First, we consider the case when $M_{2}$ is completely specified.

For each output assignment $v$, there is a corresponding set of reachable states in $S$. This results in $\left.T\right|_{\mathcal{M}(v)}$, the transition relation of the composite machine of $\left.M_{1}\right|_{\mathcal{M}(v)}, M_{2}$ and $M$. If there are different values between $o_{2}$ and $o_{M}$ in a transition of $\left.T\right|_{\mathcal{M}(v)}, G$ in Figure 5 is not a tautology. Therefore, $v$ is not a feasible output assignment. An output assignment $v$ is infeasible if and only if $\left.T\right|_{\mathcal{M}(v)}$ has a transition with $\left(o_{2} \not \equiv o_{M}\right)$. Consider a transition in $T$ as shown in Figure 6. Let the value of $h$ be $j$, and the value of $i_{2}$ be $r$. Therefore, when the output value of $e_{j}$ in $M_{1}$ is equal to that of $i_{2}$, this transition is made. State $x$ is reachable if and only if the output assignments are in $\left(\mathcal{F}_{\infty}\right)_{p=x}$. If this transition is with $\left(o_{2} \not \equiv o_{M}\right),\left(\mathcal{F}_{\infty}\right)_{p=x} \cdot\left(v_{j}=r\right)$ are infeasible output assignments. We need to enumerate all transitions of $T$ with $\left(o_{2} \not \equiv o_{M}\right)$ to compute the set of all infeasible output assignments; the set of feasible output assignments is just the complement. In the following theorem, we present an implicit enumeration method to find the feasible output assignment function, $f(v)$.

Theorem 5.3 ( $M_{2}$ : completely specified)

Let $\mathcal{W}(p, v)$ be defined as follows :

$\mathcal{W}(p, v)=\sum_{j=0}^{k-1} \theta_{i_{2}, v_{j}}\left\{\left[\exists_{n, o_{M}, o_{2}}\left(\left(\exists_{i} T\right) \cdot\left(o_{2} \not \equiv o_{M}\right)\right)\right]_{(h=j)}\right\}$.

The feasible output assignment function $f(v)$ is :

$$
f(v)=\overline{\exists_{p}\left(\mathcal{F}_{\infty} \cdot \mathcal{W}(p, v)\right)} .
$$

The feasible output assignment function $f(v)$ may be a relatively small subset of all output assignments, $V$. Moreover, it is not necessary to construct $\mathcal{F}$ first using the method in Theorem 5.1, and then remove all infeasible output assignments using Theorem 5.3. We may incrementally remove the infeasible output assignments during the construction of the reachability relation. In the next theorem, we present an incremental approach.

Theorem 5.4 ( $M_{2}$ : completely specified)

The set of feasible output assignments $f(v)$ is the limit of the following sequence, where $\mathcal{C}_{m}(p, v)$ is the reachability relation restricted to $f_{m}(v)$, the feasible output assignment function up to the $m$-th iteration. $\mathcal{P}(p, n, v)$ and $\mathcal{W}(p, v)$ are defined in Equations (1) and (2), respectively.

$$
\begin{aligned}
\mathcal{C}_{0} & =\left(p=q_{0}\right) \cdot 1, \quad f_{0}(v)=1 \\
\mathcal{C}_{m}{ }^{\prime} & =\theta_{n, p} \exists_{p}\left\{\mathcal{C}_{m-1} \cdot \mathcal{P}(p, n, v)\right\}+\mathcal{C}_{m-1} \\
\overline{f_{m}(v)} & ={ }_{p}\left(\mathcal{C}_{m}{ }^{\prime} \cdot \mathcal{W}(p, v)\right)+\overline{f_{m-1}(v)} \\
\mathcal{C}_{m} & =\mathcal{C}_{m}{ }^{\prime} \cdot f_{m}(v) \\
\mathcal{C}_{\infty} & =\mathcal{C}_{m}, \quad f_{\infty}=f_{m} \text { if } \mathcal{C}_{m}=\mathcal{C}_{m+1}
\end{aligned}
$$

In particular, $\mathcal{C}_{\infty}$ is the reachability relation restricted to $f(v)$, the feasible output assignment function.

When $M_{2}$ is input-incompletely-specified (i.e. with unspecified transitions), we need to modify the above theorem to compute $f(v)$. As explained in the previous section, input-incompletely-specified don't cares are due to the interaction with other machines (in our case, $\left.M_{1}\right)$. Let $\mathcal{L}\left(M_{2}^{i}\right)$ denote the input sequences of $M_{2}$ where the behavior is defined. We can construct an automaton $\mathcal{A}$ to accept $\mathcal{L}\left(M_{2}^{i}\right)$ as follows. Every state of $M_{2}$ is a final state. For each transition in the STG of $M_{2}$, remove the output part. Then for each state with unspecified input values, create a transition edge to the dead state (a non-final state), and associate those unspecified values to that transition. Any input sequences not in $\mathcal{L}\left(M_{2}^{i}\right)$ drive $M_{2}$ to exhibit undefined behavior. Suppose $M \equiv M_{1} \rightarrow M_{2}$ does not have undefined behavior. Then a feasible output assignment $v,\left.M_{1}\right|_{\mathcal{M}(v)} \rightarrow$ $M_{2}$ should not have undefined behavior, either. In other words, if $v$ is a feasible output assignment, the output sequences generated by $\left.M_{1}\right|_{\mathcal{M}(v)}$ must be in $\mathcal{L}\left(M_{2}^{i}\right)$ (i.e. $\left.\mathcal{L}\left(M_{1}^{o}\right)\right|_{\mathcal{M}(v)} \subseteq \mathcal{L}\left(M_{2}^{i}\right)$ ).

Theorem $5.5\left(M_{2}\right.$ : input-incompletely-specified)

The feasible output assignment function $f(v)$ can be computed using Equations (3) - (7) except that $\mathcal{W}(p, v)$ is defined as follows :

$T_{2}^{C}\left(i_{2}, p_{2}\right)=\overline{\exists_{n_{2}, o_{2}}\left(T_{2}\right)}$

$\mathcal{W}(p, v)=\sum_{j=0}^{k-1} \theta_{i_{2}, v_{j}}\left\{\left[T_{2}^{C}+\exists_{n, o_{M}, o_{2}}\left(\left(\exists_{i} T\right) \cdot\left(o_{2} \not \equiv o_{M}\right)\right)\right]_{(h=j)}\right\}$.

We can compute the feasible output assignment function $f(v)$ using Theorem 5.4 or 5.5 depending on whether $M_{2}$ is input completely specified. As an example, consider the cascade machine in Figure 4, where $M_{2}$ is completely specified. The feasible output assignments $f(v)$ can be computed by the above algorithms.

$$
\begin{aligned}
f\left(v_{1}, v_{2}, v_{3}, v_{4}, v_{5}, v_{6}\right)= & \overline{v_{1}} v_{2} \overline{v_{3}} v_{4} \overline{v_{5}} v_{6}+\overline{v_{1}} v_{2} \overline{v_{3}} \overline{v_{4}} v_{5} \overline{v_{6}} \\
& +v_{1} \overline{v_{2}} v_{3} \overline{v_{4}} v_{5} \overline{v_{6}}+v_{1} \overline{v_{2}} v_{3} v_{4} \overline{v_{5}} v_{6} .
\end{aligned}
$$

\subsection{Relationship Between Feasible Output Assign- ments and Permissible Observability Relations}

The feasible output assignment function, $f(v)$, is a multiple-valued function, thus it can be expressed in terms of a multiple-valued sumof-products.

Lemma 5.6 Let $c$ be a multiple-valued cube in the space of $V$. $\left.T_{1}\right|_{\mathcal{M}(c)}$ can be expressed using a single symbolic relation. Conversely, the output assignments of a symbolic relation $T_{1}\left(i_{1}, p_{1}, n_{1}, o_{1}\right)$ can be expressed in terms of a multiple-valued cube in the space of $V$.

Definition 6 Let c be a cube of $f(v)$, the feasible output assignment function. $\left.T_{1}\right|_{\mathcal{M}(c)}$ is called a permissible observability relation in the sense that any implementation compatible with $\left.T_{1}\right|_{\mathcal{M}(c)}$ is feasible.

Definition 7 Let $p$ be a prime of $f(v)$, the feasible output assignment function. Then $\left.T_{1}\right|_{\mathcal{M}(p)}$ is called a prime permissible observability relation in the sense that it is not contained in any other permissible observability relations.

Theorem 5.7 Let $f(v)$ be the feasible output assignment function. $\left\{\left.T_{1}\right|_{\mathcal{M}(f(v))}\right\}$ is a set of permissible observability relations.

Therefore, all feasible output functions can be expressed in terms of a set of permissible observability relations, and vice versa. By Lemma 5.6, a permissible observability relation covers some feasible output functions. Therefore, the representation for a set of permissible observability relations is not unique. The minimum set of permissible observability relations is a cover which covers all feasible output functions with the minimum number of permissible observability relations.

Theorem 5.8 The cardinality of the minimum set of permissible observability relations is equal to the cardinality of the minimum sum-ofproducts cover of $f(v)$, the corresponding feasible output assignment function. 


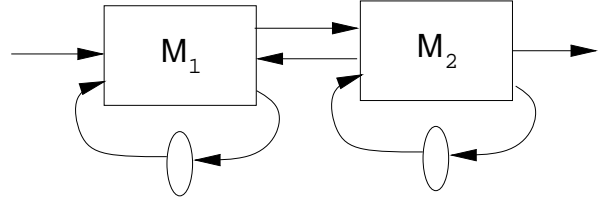

Figure 7: A two-way-communication FSM network.

In contrast, we only need to use a single observability relation, say $\mathcal{O}(i, o)$ (where $i$ is the input, and $o$ is the output), to express all the flexibility of implementation for a component in a hierarchically designed combinational logic circuit. A minterm of $i$ may map to several minterms of $o$, and it is independent of other minterms of $i$. However, this is not true in the sequential case. That is, a minterm of $i$ may map to several minterms of $o$, but it is dependent on the other minterms of $i$. Consequently, the notion of Boolean relations must be generalized for hierarchical designed sequential circuits, i.e. sets of permissible observability relations.

\subsection{Permissible Observability Relations vs. Output Don't Care Sequences}

In the next theorem, we state the relationship between the set of permissible observability relations and output don't care sequences.

Theorem 5.9 If the transition function of $M_{1}$ is not changed, and $f(v)$ is the feasible output assignment function, then the corresponding set of permissible observability relations, $\left\{\left.T_{1}\right|_{\mathcal{M}(f(v))}\right\}$, exactly captures output don't care sequences for $M_{1}$.

In a sense, our algorithms compute a class of permissible machines, and these machines can be expressed in terms of a set of permissible observability relations.

Although we have only considered one-way-communication circuits (i.e. cascade machines) so far, the above algorithms can be directly applied to compute permissible relations for a component in a two-way-communication circuit as shown in Figure 7. An interesting extension is to use these algorithms to compute permissible relations for both submachines $M_{1}$ and $M_{2}$ in Figure 7 simultaneously. We simply keep both state transition functions of $M_{1}$ and $M_{2}$ intact, and then use the above algorithms to get the feasible output function assignments of both machines simultaneously, i.e. the new output assignment space is the Cartesian product of output assignment spaces of $M_{1}$ and $M_{2}$. Note that the flexibility in implementing one submachine is dependent on that of the other. That is, their flexibility of implementation need to be compatible. With this approach, we are able to compute this compatible flexibility in implementing these submachines.

\subsection{Further Discussion}

A restriction on our algorithms is that during the computation step the state transition function of $M_{1}$ is kept unchanged, although the state transition function may change after the exploitation of output don't care information using state minimization procedures for incompletely specified FSM's. This may limit possible exploitation of other output don't care information. Therefore, the don't care information derived using our algorithms may be affected by the given state transition function of $M_{1}$. In contrast, Rho's procedure [14] employs a heuristic based on state splitting to accommodate don't care information (a subset of output don't care sequences with a fixed-length) as much as possible. Therefore, the state transition function of the component may change. This limits the exploitation of other possible output don't care information as well since the definitions of equivalent sequences in [14] is not general as explained in Section 3.3. Moreover, our algorithms implicitly enumerate infinite-length output don't care sequences using FSM equivalence checking, while Rho's procedure is limited to fixed-length sequences. Besides, our algorithms can be directly applied to handle two-way-communication circuits as shown Figure 7, and can be extended to compute compatible flexibility in implementing these two interacting components.
Consider the following case. Let $M_{1}^{\prime}$ be a machine with the same input and output alphabets as those of $M_{1}$ but with a different state space $Q_{1}^{\prime}$ and a different transition function $\delta_{1}^{\prime}$. Our algorithms can be applied to check if there exists an output function $\lambda_{1}^{\prime}$ such that $M_{1}^{\prime} \rightarrow M_{2}$ and $M_{1} \rightarrow M_{2}$ have the same I/O behavior.

Based on the above discussion, a possible approach to effectively compute and exploit output don't care sequences is as follows. Some fixed-length output don't sequences can be exploited by using state splitting. Therefore, the state transition function of $M_{1}$ changes. Infinite-length output don't care sequences can be implicitly enumerated to check if there exist better feasible output functions under this new transition function. Thus, how to perform state splitting properly such that as much output don't care information as possible can be exploited is of interest. Currently, we are investigating this problem.

\section{Exploitation of Permissible Observability Relations}

In the following, we discuss different aspects of exploiting permissible observability relations.

\subsection{State Minimization}

Limited observability may be beneficial in minimizing the number of states of a machine. A machine with the minimum number of states may not have the best implementation. However, it can be a good starting point for state assignment if the machine is not encoded yet, or for sequential logic optimization if the machine is encoded.

However, current state minimization algorithms only manipulate one observability relation (transition relation) at a time. By Lemma 5.6, a cube of $f(v)$ corresponds to a permissible observability relation. So in order to fully exploit the set of permissible observability relations, one must run state minimization procedures several times. Let $p$ be a prime of $f(v)$. Then $\left.T_{1}\right|_{\mathcal{M}(p)}$ is a prime permissible observability relation which can not be contained in any other permissible observability relations. Therefore, a state minimization procedure needs to be executed as many times as the number of primes of $f(v)$.

\subsection{Implementation}

In the case of combinational logic, a Boolean relation is sufficient to capture all the freedom of implementation. However, in the case of FSM networks, a set of permissible observability relations may be needed to represent output don't care sequences for a component. Each minterm of $f(v)$ corresponds to a feasible implementation. Now, suppose the machine is encoded. In order to find the best implementation, we need to consider every minterm of $f(v)$. From Theorem 5.8 , the minimum number of times we need to run the Boolean relation minimizer to find the best implementation is equal to the number of terms in a minimum sum-of-products for $f(v)$. The rationale is as follows : A product term in the minimized sum-of-products form of $f(v)$ corresponds to a Boolean relation, and every minterm of $f(v)$ is covered by the sum-of-products form with minimum cardinality. Consequently, we can pick the best result from all Boolean minimization executions.

\subsection{Encoding of Interconnection Signals}

Another direct application to permissible observability relations is the encoding of signals between interacting FSM's. We may convert the set of interacting binary signals between components into a symbolic variable for the purpose of re-encoding. For example, let us consider a cascade machine $M_{1} \rightarrow M_{2}$. A good output encoding of $M_{1}$ may be a bad input encoding for $M_{2}$, and vice versa. Re-encoding can be imagined as a means of moving logic between two interacting machines [6].

Shen et al. [18] give a formulation to this problem but without experimental results. They simply combine the I/O constraints from each individual component and convert them into a dichotomy covering problem with some conflict resolution techniques. Then they try to satisfy as many constraints as possible. However, this is not an exact formulation since it does not consider the exact output encoding (e.g., GPI's [7]) or any don't care information (e.g. symbolic relation). Moreover, they did not consider sequential don't cares (e.g. permissible observability relations and input don't care sequences are not used). The general formulation is still an open problem. 


\begin{tabular}{|l||r|r|r||r|r|r||r|r|r|}
\hline \multicolumn{1}{|c||}{ circuit } & \multicolumn{3}{c||}{ driving } & \multicolumn{3}{c||}{ driven } & \# fea. & \# rel. & \multicolumn{1}{c|}{$\begin{array}{c}\text { CPU } \\
\text { time }\end{array}$} \\
\cline { 2 - 8 } & I & O & S & I & O & S & & & \\
\hline \hline P & 1 & 1 & 3 & 1 & 1 & 3 & 4 & 4 & 0.1 \\
\hline bbara-bbtas & 4 & 2 & 7 & 2 & 2 & 6 & 203584 & 6 & 51.5 \\
\hline bbtas-ex5 & 2 & 2 & 6 & 2 & 2 & 8 & 2096 & 5 & 1.9 \\
\hline bbtas-ex7 & 2 & 2 & 6 & 2 & 2 & 8 & 160512 & 4 & 1.6 \\
\hline dk27-lion & 1 & 2 & 7 & 2 & 1 & 4 & 499941 & 18 & 41.1 \\
\hline ex2-ex5 & 2 & 2 & 19 & 2 & 2 & 8 & 32768 & 1 & 57.8 \\
\hline ex2-ex7 & 2 & 2 & 19 & 2 & 2 & 8 & 8704 & 2 & 20.4 \\
\hline ex3-bbtas & 2 & 2 & 11 & 2 & 2 & 6 & 81 & 1 & 4.2 \\
\hline ex3-ex7 & 2 & 2 & 11 & 2 & 2 & 8 & 12 & 1 & 7.9 \\
\hline ex5-bbtas & 2 & 2 & 8 & 2 & 2 & 6 & 243 & 1 & 2.6 \\
\hline ex5-ex7 & 2 & 2 & 8 & 2 & 2 & 8 & 128 & 1 & 3.2 \\
\hline ex3-ex5 & 2 & 2 & 11 & 2 & 2 & 8 & 1 & 1 & 10.9 \\
\hline ex2-ex3 & 2 & 2 & 19 & 2 & 2 & 11 & 1 & 1 & 20.9 \\
\hline
\end{tabular}

Table 1: Experimental results

$\begin{array}{ll}\text { S : } & \text { number of states in isolation } \\ \text { \# fea. : } & \text { number of feasible output assignments of the driving machine } \\ \text { \# rel. : } & \text { minimum number of permissible observability relations } \\ \text { CPU time : } & \text { in seconds on a DEC 5000/260 with } 128 \mathrm{MB}\end{array}$

Permissible observability relations allow us to have many feasible output functions, and in theory these should be useful for encoding the interacting signals.

\section{Experimental Results}

In this section, we present some preliminary results. Most of the examples are obtained by interconnecting FSM's from the MCNC benchmarks. These FSM's are completely specified and state-minimal in isolation. Example P is shown in Figure 4.

Table 1 shows the results of some cascade circuits consisting of two FSM's. The minimum number of permissible relations is obtained using ESPRESSO-MV [16]. The CPU time indicated includes both the computation of feasible output assignments and ESPRESSOMV. Although the feasible output functions of examples ex2-ex5, ex3-bbtas, ex3-ex7, ex5-bbtas and ex5-ex7 can be expressed by a single relation individually, they cannot be computed using either Devadas' or Rho's procedures. In examples bbara-bbtas, $\mathrm{P}$, bbt as-ex 5, bbtas-ex 7, dk27-lion and ex2-ex 7, the minimum number of permissible relations to express the feasible output functions is more than one. The number of feasible output functions in examples ex3-ex5 and ex2-ex3 is one.

Our implicit algorithm based on BDD's deals with all output assignments at a time. With our current implementation, we can handle small-size examples in a reasonable amount of CPU time as shown in Table 1. In contrast, the explicit algorithm which enumerates all possible output assignments one by one is very inefficient since the number of all output assignments is too large. For instance, there are $2^{152}$ possible output assignments in example ex $2-e \times 7$, but only 8704 of them are feasible. The feasible output assignment function, $f(v)$, is normally a relatively small subset of $V$, the set of all output assignments. Therefore, proper BDD variable ordering or use of 0-Sup-BDDs [12] should enhance the ability and efficiency of our algorithms. Currently, we are studying a good BDD variable ordering to handle larger examples.

The output part of a transition edge in a permissible relation is a multiple-valued literal. As pointed out in [6], pairwise compatibility of a set of states $S$ does not imply $S$ is compatible. Thus, additional checking has to be performed during state minimization. At the present time, there are no state minimization programs with this ability in our logic synthesis system.

\section{Conclusion}

We discussed intrinsic difficulties in computing output don't care sequences for a component in an FSM network. We pointed out that these can not be explained using information lossyness [14]. We demonstrated that output don't care sequences for a component can be expressed using a set of permissible observability relations given that its state transition function is kept unchanged. We presented a novel approach to exactly compute them. The representation for a set of permissible observability relations is not unique. We provided a method to find a set with the minimum number of permissible observability relations. We also discussed the applications of permissible observability relations in different contexts, such as state minimization, circuit implementation and signal encoding.

\section{Acknowledgements}

The authors are thankful to Dr. June-Kyung Rho for providing valuable information. We thank Dr. Rajeev Murgai for reading and improving this manuscript. Also special thanks to Szu-Tsung Cheng and Thomas Shiple for helpful discussions on the BDD package.

\section{References}

[1] R. K. Brayton and F. Somenzi. Boolean Relations and the Incomplete Specification of Logic Networks. In VLSI'89, August 1989.

[2] R. E. Bryant. Graph Based Algorithms for Boolean Function Manipulation. IEEE Transactions on Computers, C-35(8):677-691, August 1986.

[3] E. Cerny and M. A. Marin. An Approach to Unified Methodology of Combinational Switching Circuits. In IEEE Transactions on Computers, pages 745-756, August 1977.

[4] O. Coudert, C. Berthet, and J. C. Madre. Verification of Sequential Machines Based on Symbolic Execution. In Proceedings of the Workshop on Automatic Verification Methods for Finite State Systems, Grenoble, France, 1989.

[5] M. Damiani and G. De Micheli. Recurrence Equations and the Optimization of Synchronous Circuits. In 28th ACM/IEEE Design Automation Conference, pages 556-561, June 1992.

[6] S. Devadas. Optimizing Interacting Finite State Machines Using Sequential Don't Cares. In IEEE Transactions on Computer Aided Design of Integrated Circuits and Systems, pages 1473-1484, December 1991.

[7] S. Devadas and A. R. Newton. Exact Alogrithms for Output Encoding, State Assignment and Four-level Boolean Minimization. In IEEE Transactions on Computer Aided Design of Integrated Circui ts and Systems, pages 13-27, January 1991.

[8] G. Hachtel, J. K. Rho, F. Somenzi, and R. Jacoby. Exact and Heuristic Algorithms for the Minimization of Incompletely Specified State Machines. In The European Conference on Design Automation, 1991

[9] J. Kim and M. M. Newborn. The Simplification of Sequential Machines With Input Restrictions. In IEEE Transactions on Computers, pages 1440-1443, December 1972.

[10] B. Lin and F. Somenzi. Minimization of Symbolic Relations. In IEEE International Conference on Computer-Aided Design, pages 88-91, November 1990.

[11] B. Lin, H. Touati, and A. R. Newton. Don't Care Minimization of Multi-Level Sequential Logic Networks. In IEEE International Conference on Computer-Aided Design, pages 414-417, November 1990.

[12] S. Minato. Zero-SuppressedBDDs for Set Manipulation in Combinatorial Problems. In 30th ACM/IEEE Design Automation Conference, pages 272-277, June 1993.

[13] M. C. Paull and S. H. Unger. Minimizing the Number of States in Incompletely Specified Sequential Circuits. In IRE Transactions on Electronic Computers, pages 356-366, September 1959.

[14] J. K. Rho, G. Hachtel, and F. Somenzi.Don't Care Sequences and the Optimization of Interacting Finite State Machines. In IEEE International Conference on ComputerAided Design, pages 418-421, November 1991.

[15] J. K. Rho, G. Hachtel, and F. Somenzi. Don't Care Sequences and the Optimization of Interacting Finite State Machines. In International Workship on Logic Synthesis, May 1991.

[16] R. Rudell and A. Sangiovanni-Vincentelli. Multiple-Valued Minimization for PLA Optimization. In IEEE Transactions on Computer Aided Design of Integrated Circuit and Systems, pages 727-750, 1987.

[17] H. Savoj and R. K. Brayton. Observability Relations and Observability Don't Cares. In IEEE International Conference on Computer-Aided Design, pages 518-521, November 1991.

[18] J. J. Shen, Zafar Hasan, and M. J. Ciesielski. State Assignment for General FSM Networks. In The European Conference on Design Automation, pages 245-249, 1992.

[19] H.-Y. Wang and R. K. Brayton. Input Don't Care Sequences in FSM Networks. In IEEE International Conference on Computer-Aided Design, pages 321-328, November 1993.

[20] H.-Y. Wang and R. K. Brayton. Permissible Observability Relations in FSM Networks. Technical Report UCB/ERL M94/15, University of California, Berkeley, February 1994. 